\title{
Mafia, Militär und »tiefer Staat «- Verschwörungstheorien und Realitäten in »Kurtlar Vadisi Pusu«
}

\author{
Sara Merdian
}

\section{$1 \quad$ Einleitung und Hintergründe zu Kurtlar Vadisi}

Zum Saisonstart im Herbst wartet das türkische Fernsehen jeweils mit einer Reihe neuer Serien auf, die oft in kurzer Zeit ein Millionenpublikum begeistern können. Unter den Erfolgsserien der letzten Jahre sind unter anderem Verfilmungen von Literaturklassikern wie etwa »Aşk-i memnu « oder » Yaprak Dökümü«, Erzählungen aus dem Leben junger Großstädter (»Kavak Yelleri«, »Küçük Sirlar«), Historienfilme (»Muhteşem Yüzyıl «) oder auch Serien, die sich mit der türkischen Alltagsgeschichte und Politik (»Öyle bir geçer zaman ki «) auseinandersetzen. ${ }^{1}$ Erstaunlich ist dabei, dass diese Serien nicht nur das türkische Publikum allabendlich vor die TV-Geräte locken oder etwa im Internet (Youtube, Facebook) für Gesprächsstoff sorgen: auch international begeistern diese Serien simultan übersetzt oder mit Untertiteln versehen Millionen Fernsehzuschauer auf dem Balkan und in Griechenland sowie rund um das Mittelmeer und die Golfregion. Einige Beobachter dieses Phänomens sehen daher in den Serien auch einen Beitrag zur neuen türkischen Soft Power-Diplomatie, mit der das Image einer »neuen Türkei «, die islamische Tradition und westliche Moderne vereint, geschaffen werden soll. ${ }^{2}$

Eine Erfolgsgeschichte besonderer Art ist der "phänomenale « Serien-Mehrteiler »Kurtlar Vadisi« (»Tal der Wölfe«) gewesen. Die politische Actionserie begeisterte in der Türkei und darüber hinaus ein Millionenpublikum und verkaufte sich gewinnbringend in viele Länder, vor allem im Nahen und Mittleren Osten. ${ }^{3}$

1 Die genannten Serientitel lassen sich übersetzen als »Verbotene Liebe«, »Blätterfallen «, »Pappelwinde «, » Kleine Geheimnisse «, »Ein wunderbares Jahrhundert « bzw. » Wie die Zeit vergeht «.

2 Vgl. »TV-Serien: neue diplomatische Kraft der Türkei «. Deutsch-Türkische Nachrichten, 12. Dezember 2011.

3 Vgl. »Polat Alemdar Suriye'de fenomen oldu «. Habertürk, 19. September 2012. 
Das Geheimnis ihres Erfolges liegt vor allem in ihrer stark von Action-Szenen dominierten Umsetzung, die im Wesentlichen darauf abzielen, reale politische Entwicklungen der jüngeren Vergangenheit in der Türkei und ihrer regionalen Nachbarschaft skandalisierend abzubilden. Dabei erhebt die Serie den Anspruch klandestine und mafiöse Machenschaften aus der realen Welt der Politik fiktional » aufdecken « zu wollen. ${ }^{4}$ Sie stellt daher einen interessanten Forschungsgegenstand dar, der jedoch bislang nur wenig und unsystematisch mit Blick auf seine Inhalte, Machart und Wirkungsweise untersucht wurde. Bevor auf diese Aspekte näher eingegangen werden kann, werden noch einige Hintergrundinformationen zur Verlaufsgeschichte der Produktion der Serie und ihrer " Macher " gegeben.

Im ersten, sehr erfolgreichen Teil der Serie »Kurtlar Vadisi «, die von 2003 bis 2005 auf dem türkischen Kanal Show TV lief, war der Schauspieler und Hauptdarsteller Necati Şaşmaz als türkischer Geheimagent zu sehen, der als verdeckter Ermittler in die Mafia eingeschleust wird und sich dazu eine neue Identität zulegt. In der Rolle des Polat Alemdar stellte Şaşmaz einen Agenten dar, der mit seinen Agentenkollegen Memati Baş (Gürkan Uygun) und Abdülhey Çoban (Kenan Çoban) die türkische Mafia zerschlagen sollte. Die Fortsetzung der Serie, die unter dem Titel »Kurtlar Vadisi Terör « 2007 anlief, wurde trotz erneut hoher Einschaltquoten schon nach zwei Folgen wegen unsachlicher Darstellung realer politischer Ereignisse vom Obersten Rat für Hörfunk und Fernsehen (Radyo ve Televizyon Üst Kurulu, RTÜK) abgesetzt. ${ }^{5}$ Zuvor hatte bereits 2006 der Kinofilm »Kurtlar Vadisi Irak« (»Tal der Wölfe: Irak«) in der Türkei ebenso wie in Europa und im Nahen und Mittleren Osten ein Massenpublikum in die Kinos gelockt. 2007 wurde die Serie unter dem Titel »Kurtlar Vadisi Pusu« (»Tal der Wölfe: Hinterhalt «) fortgesetzt und wird bis heute mit Erfolg in der siebten Staffel ausgestrahlt. Sie steht im Zentrum dieses Beitrages, weil sie sich wie kein anderer Teil der Serie dafür eignet, um zentrale Erzählelemente zu analysieren und auf ihre Rückkopplungen mit den real ablaufenden politischen Entwicklungen hinzuweisen.

Alle Teile der Serie sowie ihre weiteren Spin-Offs ${ }^{6}$ wurden von der Filmgesellschaft Panafilm produziert, in der der Filmemacher Racı Şaşmaz, der Regisseur Zübeyr Şaşmaz und der Hauptdarsteller der Serie Necati Şaşmaz zusammenarbeiten. Sie alle sind Kinder von Şeyh Abdülkadir Şaşmaz, der dem Sufi-Orden

4 Vgl. Panafilm 2009.

5 Vgl. Kürsü 2007. Da dieser Teil der Serie sich mit den politischen Verhältnissen in der Südosttürkei auseinandersetzen sollte, ist anzunehmen, dass eine Darstellung und Interpretation des dort herrschenden Kurdenkonflikts zu heftigen Reaktionen hätte führen können.

6 "Kurtlar Vadisi Gladio" (»Tal der Wölfe: Gladio «, 2009), "Muro: Nalet olsun içimdeki insan sevgisine (»Muro: Verflucht sei die Menschenliebe in mir «, 2008) und »Kurtlar Vadisi Filistin « (»Tal der Wölfe: Palästina «, 2011). 
der Kadiri vorsteht. ${ }^{7}$ Über das Netzwerk des Kadiri-Ordens und die weiteren verwandtschaftlichen Verflechtungen der Familie Şaşmaz, die etwa auch in die islamisch-nationalistische Milliyetçi Hareket Partisi (MHP, „Partei der Nationalistischen Bewegung ") reichen, lassen sich die Panafilm-Produktionen ideologisch einordnen. So spiegelt nicht nur die Serie insgesamt nationalistische und islamische Motive, Vorstellungen und Wahrnehmungen der Realität wider, sondern auch das Umfeld und die Biographien derer, die bei Panafilm involviert sind. ${ }^{8}$

\section{2 "Kurtlar Vadisi Pusu «: Fiktion und Realität am Beispiel der »Ergenekon-Verschwörung"}

Wie schon in den ersten Teilen der Serie spielt auch in dem seit 2007 laufenden Serienteil »Kurtlar Vadisi Pusu « die kriminelle Unterwelt der Türkei eine zentrale Rolle. Der Einfluss dieser Unterwelt auf aktuelle politische Ereignisse in der Türkei wird dabei ebenso thematisiert wie der andauernde Kurdenkonflikt. Die Serie erzählt von reichen Geschäftsleuten, korrumpierten Militärs und ihren Versuchen - im Namen unterschiedlicher Ideologien -, Einfluss auf die türkische Politik zu nehmen. Darüber hinaus ist die Serie stark mit Verschwörungstheorien darüber angereichert, wie »Amerika « die türkische Innenpolitik beeinflusst. So wird zwar angenommen, dass sich ein Teil der gewählten Repräsentanten des Volkes sowie viele hochrangige Militärs für einen Frieden zwischen den ethnischen Gruppen im Land einsetzen. Doch zugleich wird - wie unten zu sehen sein wird "die Wahrheit « hervorgehoben, dass eine mehr oder weniger geheime Gruppe von Politikern und Militärs das Land im Austausch für Macht und Reichtum an die Amerikaner verkauft haben.

Den politisch interessierten Zuschauer und informierten Beobachter der Serie mag auf den ersten Blick überraschen, mit welcher Nonchalance die Produzenten der Serie zwischen Action-reicher Fiktion und suggestiver Realität hin- und herwechseln. Manchmal sind es nur nebensächliche Details, die Auskunft über die Aktualität und Realität der Serie geben, wie etwa an einer Stelle der Hinweis auf eine massive Vulkaneruption auf Island, die mit dem realen Ausbruch des Eyjafjallajökull im April 2010 korrespondiert, oder auch von den Akteuren geäußerte Einschätzungen zu der sich stabilisierenden Wirtschaftslage in der Türkei. Die zentralen Themen der Serie sind jedoch die großen politischen Ereignisse, Konflikte und Skandale im Land. Der Machtkampf zwischen politischen Eliten und

7 Vgl. Kurt 2004, Satmış 2004.

8 Vgl. Rebhandl 2010. 
Ideologien wird dabei ebenso thematisiert wie die organisierte Kriminalität, Korruption und gesellschaftliche Spannungen zwischen den verschiedenen sozialen, ethnischen und religiösen Gruppen in der Türkei. ${ }^{9}$

Dabei ist diese Nähe zur Realität ein geschickt eingesetztes Mittel, um die $\mathrm{Zu}$ schauer an die fiktive Story zu fesseln und ihnen eine - wie unten gezeigt wird zugegeben recht simple - Interpretationshilfe zum besseren Verständnis der politischen und gesellschaftlichen Realität an die Hand zu geben. Gleichzeitig steht die Serie durch diesen engen Aktualitätsbezug auch unter einem enormen Druck sich zu verändern, sich an die sich wandelnden realen Gegebenheiten anzupassen und neue Interpretationen zu politischen Ereignissen zu liefern. Kann sie nicht mit interessanten Erkenntnissen aufwarten oder nimmt der Anteil an Action und die Eliminierung vieler Charaktere (immer gefolgt vom Eintritt neuer Akteure) überhand, so verliert sie an aussagekräftigem Inhalt und dadurch an Attaktivität für bestehende und potenzielle Zuschauer. ${ }^{10}$

Um der zentralen Frage nach dem spannungsreichen Verhältnis von Fiktion und Realität in "Kurtlar Vadisi « systematisch nachzugehen, möchte ich mich im Folgenden auf die dritte Staffel von »Kurtlar Vadisi Pusu « konzentrieren, die von 9. Oktober 2008 bis 11. Juni 2009 auf Show TV ausgestrahlt wurde. In dieser Staffel nahmen die Panafilm-Produzenten indirekt Bezug auf die so genannte Ergenekon-Verschwörung, deren Aufdeckung $2007 \mathrm{zu}$ einem politischen Erdbeben in der Türkei geführt hatte. Dessen Auswirkungen dauern in Form des juristischen und soziopolitischen Aufarbeitungsprozesses bis heute an. Bei der Ergenekon-Verschwörung selbst handelt es sich im Kern um Putschpläne von Teilen des Militärs und verbündeter Gruppen und Organisationen gegen die »islamistische» Regierung der Adalet ve Kalkınma Partisi (AKP), deren Zweck es war, die laizistisch-nationalistische Ordnung der Türkei aufrecht zu erhalten. Im Rahmen der bisherigen juristischen Untersuchungen wurden erstmals hochrangige Militärs festgenommen und vor Zivilgerichte geladen sowie Strukturen und Machtausübung der Armee in Frage gestellt. ${ }^{11}$

9 Vgl. dazu allgemein Hermann 2008, Kramer 2009b. Um die durchlässige Trennlinie zwischen Fiktion und Realität einigermaßen aufrecht zu erhalten, sind in diesem Essay fiktionale Erscheinungen der Serie in Anführungszeichen gesetzt, während diese bei den realen Erscheinungen fehlen.

10 Anders als hier gezeigt, gab es allerdings auch Fälle dynamischer Seriennarration, die den nachhaltigen wirtschaftlichen Erfolg offenbaren: So machten sich die Produzenten etwa die usprünglich nicht intendierte positive Resonanz unter den Zuschauern auf den ins Drogen- und Organgeschäft verwickelten PKK-Aktivisten Muro, der sich im Verlauf der Serie zu einer positiven Gestalt mit Idealen und Überzeugungen mauserte, zu Nutze und brachten 2008 einen Kinofilm mit » Muro « als Protagonisten heraus. Vgl. Özuzcan 2012.

11 Vgl. dazu Hermann 2008, S. 233 ff., Kramer 2009b. 
Bei der Interpretation dieser Staffel möchte ich zum einen auf die Darstellungen in der Serie ${ }^{12}$ und zum anderen auf belegte Hinweise rund um den Ergenekon-Prozess (und die im Zusammenhang stehende Vorgeschichte) zurückgreifen. Diese Gegenüberstellung steht in den folgenden Abschnitten im Vordergrund. Allerdings soll es nicht nur um die theoretische Frage »Fiktion vs. Realität « im Hinblick auf "Kurtlar Vadisi Pusu « gehen, sondern auch um die empirische Frage, ob und wenn, wie sich die Wahrnehmung der Serie, ihrer Inhalte und Themen durch die Zuschauer im Verlauf der Serie gewandelt hat. Dafür habe ich im Winter 2008 und im Sommer 2010 in der Türkei eine nicht-repräsentative Befragung unter Studenten und Zuschauern der Serie geführt. ${ }^{13}$ Gegenstand dieser Interviews waren sowohl Erwartungen an die politische Entwicklung des Landes als auch Möglichkeiten der politischen Partizipation. Ein vielfach beobachtetes Moment unter den Befragten war dabei das Verhältnis von politischer Realität und Fiktion in der Serie. Unter ihnen war festzustellen, dass die Zahl der »Fans « der Serie von 2008 bis 2010 stark abgenommen hatte. Viele hatten sich offenbar gelangweilt von der Serie abgewandt oder verfolgten sie nur noch sporadisch. Hatte also die politische Realität die fiktionale Serie an Spannung überholt? Oder hatte sich die Wahrnehmung und politische Erwartungshaltung der Zuschauer an Fiktion und Realität in den zwei Jahren so dramatisch verändert, dass die Serie mit ihrem interpretativen Anspruch an Einfluss verloren hatte? Ließen sich also diese veränderte Wahrnehmung und der schwächer werdende Glaube an Verschwörungstheorien mit der wachsenden demokratischen Haltung der Menschen in der Türkei erklären?

Um diese Fragen am Beispiel der Ergenekon-Verschwörung als Serienthema und realpolitischer Entwicklung zu beantworten, werden zunächst die Serie und ihre Darstellung von Staat und Politik näher betrachtet. Anhand der fiktionalen Vorstellung vom real existierenden »tiefen Staat « und vor dem Hintergrund der neo-nationalistischen Bewegung in der Türkei wird herausgearbeitet, wie die Serie die Ergenekon-Verschwörung darstellt und auf welche ideologischen Muster bei der Darstellung und Interpretation zurückgegriffen wird. Vor diesem Hintergrund wird das sich verändernde Bild, das sich junge Menschen vom türkischen Staat machen, erläutert und die von mir angenommene Veränderung dieser zunehmend sachlichen Wahrnehmung von Politik näher betrachtet.

12 In einigen anderen Aspekten möchte ich den Blick auch auf den Film »Kurtlar Vadisi Gladio " aus dem Sommer 2009 werfen, in dem die Panafilm-Produzenten direkt auf einige reale Ereignisse im Kurdenkonflikt und auf die ihrer Meinung nach damit verbundenen Machenschaft des Staates Bezug nehmen.

13 Dabei handelte es sich um zwei Studentengruppen in Istanbul mit zehn bzw. fünf Mitgliedern, zum einen aus einem linkspolitischen, anti-kemalistischen und zum anderen aus dem muslimischen Milieu, die sich regelmäßig trafen, um aktuelle politische Themen zu diskutieren. 


\subsection{Der tiefe Staat: Realität und Fiktion}

$\mathrm{Zu}$ Beginn einer Auseinandersetzung mit »Kurtlar Vadisi Pusu « muss zunächst betont werden, dass die Art und Weise, wie politische Ereignisse in der Serie erzählt werden, selten eindeutig zu interpretieren ist. Um ein Verständnis für die Serie zu gewinnen ist es daher hilfreich, plausible Parallelen zwischen Charakteren der Serie und realen Personen zu ziehen bzw. auf Hinweise der Produzenten und Schauspieler zurückzugreifen - selbst wenn Übereinstimmungen nie eindeutig bestätigt werden. So antwortete etwa der Hauptdarsteller Necati Şaşmaz in einem Interview 2009 auf die Frage, ob sein Gegenspieler in der Serie, der Agent İskender Büyük, in der Realität General Veli Küçük, einem Hauptverdächtigen im Ergenekon-Prozess entspreche:

»Kurtlar Vadisi yalın halindeyken mafyayı anlatıyordu. Mafyayı anlatırken de `Şu şuna benziyor, bunu buna benziyor` deniliyordu. Kurtlar Vadisi Pusu'nun içerisinde de diş mihrapların yönlendirilmesiyle oluşan güç odakları olan köklü aileler vardı. O aile >Bu mu, şu mu< diye tartışmalar olmuştu. Polat için >Abdullah Çatlı mı? ‘ diyorlardı. >Avrupa'daki Çakal mı, oo7 mi? [...] Birçok karakteri birleştirip senaristlerimiz bir şey yazıyor. Nasıl geçmişte, hikayelerde bir şeyler anlatılırken asland, tavşand, kurttu, tilkiydi diye örneklendiyse günümüzde de Polat'ın, İskender'in, Ömer Baba'nın hepsinin sembolize ettiği değerler var. ${ }^{14}$

(»In seiner Originalversion erzählte Kurtlar Vadisi von der Mafia. Als wir von der Mafia erzählten, wurde auch gesagt: 〉Jener erinnert an jenen, dieser an diesen`. In Kurtlar Vadisi Pusu gab es wichtige Familien, die das Ziel von Kräften waren, die vom Ausland gelenkt wurden. Es wurde diskutiert, ob jene Familie, diese sei oder jene. Sie sagten: >Ist Polat Abdullah Çatlı? \Ist er der europäische Schakal oder oo7? « [...] Unsere Drehbuchautoren schreiben einige Charaktere zusammen. So wie früher etwas in Geschichten erzählt wurde und es einen Löwen gab, einen Hasen, einen Wolf, einen Fuchs, so stehen Polat und Ömer baba heute auch für bestimmte Werte. «)

Şaşmaz' zuletzt gemachter Vergleich von »Kurtlar Vadisi « mit einer Fabel ist bemerkenswert und löst bestimmte Erwartungen aus. Schließlich zeichnen sich Fabeln als fiktive Erzählungen dadurch aus, dass sie wahre Konflikte nachzeichnen, in denen Tiere mit menschliche Charakterzügen und Persönlichkeiten die Hauptrolle übernehmen. Dabei wird dem Leser stets eine moralische Lehre für das alltägliche, reale Leben vermittelt. Demnach möchte also auch »Kurtlar Vadisi « »Wahres« über Menschen und die Realität vermitteln. Doch welches sind diese 
Wahrheiten, über die die Serie über die reale Menschen- und Persönlichkeitsebene hinaus die Zuschauer informieren und aufklären will?

Auch hierfür dient Şaşmaz' Zitat als geeignete Quelle. Denn während er mit dem »Schakal « und »oo7 « auf zwei weitere fiktive - und damit hier vernachlässigbare - Agenten verweist, nennt er mit Abdullah Çatlı auch eine real existierende Person, die ihrerseits zur Hauptfigur eines der größten Polit-Skandale in der jüngeren türkischen Vergangenheit wurde: Nachdem am 3. November 1996 nahe der Kleinstadt Susurluk in der Nordwesttürkei ein schwarzer Mercedes mit einem Lastwagen kollidierte und drei der vier Autoinsassen starben, kam heraus, dass unter den Toten neben einem hohen Polizeioffizier auch jener Abdullah Çatlı, ein international gesuchter Krimineller, und seine Geliebte gewesen waren. Der vierte Insasse, ein Parlamentsabgeordneter, überlebte in dem mit Waffen und falschen Papieren gefüllten Pkw. ${ }^{15}$ Der Unfall oder vielmehr die Zusammensetzung dieser »Reisegruppe« sowie ihr verdächtiges Gepäck lösten viele Fragen in der Türkei aus, etwa was ein gewählter Volksrepräsentant zusammen mit einem international gesuchten Verbrecher zu tun habe und wer in der Armee ihm zu seiner falschen Identität verhalf. Der Vorfall sollte nie aufgeklärt werden, stattdessen starb sogar ein Parlamentarier, der mit der Aufklärung der Zusammenhänge beauftragt war, später unter ungeklärten Umständen. Erstmals war aber offensichtlich geworden, dass es in der Türkei Strukturen gab, in denen Politik und staatliche Sicherheitsbehörden mit der organisierten Kriminalität kollaborierten.

In der Folge führten der Vorfall und die sich daran entzündende öffentliche und mediale Diskussion nicht nur dazu, dass nun auch andere Ereignisse plötzlich aus einem neuen Blickwinkel betrachtet wurden. ${ }^{16}$ Vielmehr schien nun auch offenkundig zu werden, weshalb alle bisherigen Versuche solchen Vorfällen nachzugehen, immer im Sand verlaufen waren und die Drahtzieher nie belangt und dingfest gemacht werden konnten: Der Schluss lag nahe, dass es neben dem etablierten politischen System einen real existierenden »Staat im Staat « oder »tiefen Staat « (»derin devlet «) in der Türkei gibt, als dessen operatives Organ ein geheimes Netzwerk namens Ergenekon gilt, das aus staatlichen und nicht-staatlichen, legalen und illegalen Akteuren besteht. ${ }^{17}$

15 Vgl. Kaya 2009, S. 100, Jung 2011, S. 2.

16 Der Anschlag auf Papst Johannes Paul II. 1981, der Mord am Unternehmer Özdemir Sabanc1 1996 und am armenischen Journalisten Hrant Dink 2007 werden heute als Machwerk dieser mafiösen Strukturen angesehen. (Vgl. Kavakci 2009, S. 87.)

17 Kaya 2009 und Gingeras 2010 führen die Existenz dieses »tiefen Staates « auf die Teşkilât-ı Mahsusa (Spezialorganisation) zurück, eine geheime Gruppe, die im Osmanischen Reich vom Komitee für Einheit und Entwicklung (İttihâd ve Terakkî Cemiyeti) ins Leben gerufen worden war und, um 1913 die Machtergreifung und -erhalt des Komitees zu sichern, auch politische Gegner beseitigte. Nach Kaya und Gingeras zeigt sich an diesem frühen historischen 
In dem oben erwähnten Interview suggeriert Şaşmaz also einen direkten Bezug zwischen der fiktiven Handlung in "Kurtlar Vadisi « und dem real existierenden »tiefen Staat « stellt sogar einen Zusammenhang zwischen sich selbst in seiner Rolle als Polat Alemdar und Abdullah Çatlı her: So ist Alemdar der Protagonist im halb-legalen, lebensgefährlichen Spiel zwischen den Mächtigen in der schmutzigen türkischen und internationalen Politik. Zusammen mit seinen Kollegen Memati Baş und Abdülhey Çoban stellt er das operative Agententeam einer ihrem Selbstbild nach nicht-offiziellen, aber an Legitimität jeder staatlichen Organisation gleichberechtigten, wenn nicht sogar überlegenen Organisation namens "Kamu Güvenliği Teşkilatı (»Öffentliche Sicherheitsorganisation «, KGT). Diese wiederum arbeitet zwar selbstständig, erhält in der Serie dennoch auch Befehle und Anleitung von einer anderen im Hintergrund agierenden Organisation, den so genannten »İhtiyarlar « (»die Alten«), die ihrerseits unabhängig von der staatlichen Politik agieren und sich - wie Polat - nur für »das Gute « einsetzen..$^{18}$ Insgesamt ergibt sich so zunächst das Bild einer im Geheimen und damit abseits von staatlicher und gesetzlicher Kontrolle agierenden, quasi verbrecherischen Organisation und ihrer Agenten, die Teil eines »tiefen Staates « sind. Zu ihren Aufgaben gehört also vor allem das Eliminieren von Feinden hinter dem Rücken von Polizei, Justiz und Öffentlichkeit. Dabei können sie auf persönliche Allianzen mit staatlichen Sicherheitsorganen bauen - »Susurluk « in Reinform.

Allerdings ist es mit Blick auf die Frage nach den Auswirkungen der Realitätsrekonstruktion in "Kurtlar Vadisi Pusu « auf die türkischen Zuschauer von großer Bedeutung, darauf hinzuweisen, dass die Serie die so dargestellten Strukturen des »tiefen Staates « nicht kritisiert. Stattdessen wird die Existenz des tiefen Staates durch Staatsfeinde im In- und Ausland gerechtfertigt. Wer oder was mit diesen Feinden »konkret " gemeint ist, verdeutlicht folgender Dialog der beiden Weggefährten Alemdars, Erhan und Hikmet. Dabei geht es um eine andere tiefstaatliche Organisation, ein Netzwerk namens »Gladio «, welches der »KGT « feindlich gegenüber steht und von ihr bekämpft wird:

Hikmet: »Latince kısa kılıç demek. Amerika II dünya savaşı sonrasında özellikle Avrupayi komünist tehlikeye karsi örgütledi. Bu komünistler sizi işgal edecekler sizinde

Beispiel die instrumentelle Nähe organisierten Verbrechens und staatlicher Macht in der Türkei. Auch wenn der »tiefe Staat « und das »Ergenekon-Netzwerk « heute um einiges komplexer ist, zeige sich - so auch Jung - immer wieder »die autoritäre Traditionslinie des türkischen Modernisierungsprozesses mit den Interessen einer Koalition anti-demokratischer Kräfte aus Militär, Geheimdienst, Justizapparat, paramilitärischen Milizen sowie dem organisierten Verbrechen «. (Jung 2011, S. 2.)

18 Auch an dieser Stelle fabulieren die Serienproduzenten mit der Realität und stellen eine offenbare Parallelität zwischen der fiktiven » KGT « und der historischen Teşkilât-ı Mahsusa her. 
buna direnecek, bunu engelleyecek silahlı gücünüz yok deyip her ülkenin karar mercilerinde kendi yapılanmalarını oluşturdular bunun İtalyadaki adı gladyo. «

Erhan: »Türkiyedeki adı?»

Hikmet: »12 eylülden önce kontrguerilla dendi şimdiki ismi gazetelerde okuyorsun işte ama kesin bir isim deşifre edilmedi çünkü yapılanma deşifre edilmedi. «19

(Hikmet: »Auf Latein bedeutet [Gladio] >Kurzschwert`. Nach dem Zweiten Weltkrieg hat Amerika vor allem Europa gegen die kommunistische Gefahr organisiert. Sie sagten, die Kommunisten werden euch besetzen, ihr habt nicht genug militärische Macht, um Widerstand zu leisten, eine Besatzung zu verhindern. Und so schufen sie an den Entscheidungsorten aller Länder ihre eigenen Organisationen. In Italien heißt diese Organisation Gladio."

Erhan: »Und der Name der Organisation in der Türkei? «

Hikmet: »Vor dem 12. September [1980] wurde sie Konterguerilla genannt, ihren heutigen Namen liest du ja in der Zeitung. Ein bestimmter Name wurde jedoch nie dechiffriert, da die Organisation selbst nie dechiffriert wurde.«)

Gladio steht in der Serie also für die Versuche der USA, die Türkei (sowie damit auch den Nahen und Mittleren Osten) unter ihre Kontrolle zu bringen. Damit haben die Produzenten erneut eine direkte Verbindung zur realen historischen Vergangenheit der Türkei geschaffen. Sie erklären die Existenz » zwielichtiger Strukturen « oder gar eines »tiefen Staates « mit den so genannten stay-behind-operations der USA bzw. der NATO während des Kalten Krieges, mittels derer der Kommunismus aus den Ländern Mittel- und Südeuropas - auch mit illegalen Mitteln -

19 »Kurtlar Vadisi Pusu«, 3. Staffel 9. Oktober 2008 bis 11. Juni 2009, Episode 61, Zeit: 59:2501:02:02. An einer anderen Stelle erklärt Hikmet seinem Kameraden Erhan auch, dass »Gladio « keine militärisch-staatliche Organisation sei, sondern wie die »KGT « über tiefenstaatliche Netzwerkstrukturen verfüge:

Hikmet: »Komünizm gelecek diye dernekler kuruldu sivil toplum örgütleri oluşturuldu, medya yaratıldı, yeni iş adamları ortaya çıkarıldı ve tabiki siyasetçiler yetiştirildi. « (»Unter dem Vorwand, dass der Kommunismus kommen werde, wurden Vereine gegründet, zivilgesellschaftliche Organisationen entstanden, Medien wurden geschaffen, neue Geschäftsleute wurden eingesetzt und natürlich wurden Politiker herangezogen. «) ("Kurtlar Vadisi Pusu «, 3. Staffel 9. Oktober 2008 bis 11. Juni 2009, Episode 61, Zeit: 26:34-26:44.) Zudem treten immer wieder Vertreter »Gladios « auf, die Polat anbieten, er könne die Türkei beherrschen oder gar die ganze Region, wenn er sich »Gladio « verpflichte - doch Polat bleibt freilich standhaft und stellt sich hinter die Sache, für die er kämpft. (Vgl. ebd.) 
zurückgedrängt werden sollte. Auch wenn mittlerweile die Geschichtsforschung erste Ergebnisse zu diesen realen Operationen vorlegen konnte, ${ }^{20}$ dominiert etwa in der Türkei in diesem Bereich noch verschwörungstheoretisches »Wissen «. So verweist etwa auch Jenkins auf den türkischen Journalisten Erol Mütercimler, der im Rahmen der Nachforschungen zum Unfall in Susurluk behauptete, er habe Informationen darüber, wonach die US-amerikanische CIA eine Geheimorganisation namens Ergenekon nach 1960 in der Türkei aufgebaut habe. In ihrer Nachfolge sollen, so Mütemcimler, auch heute noch Organisationen tätig sein, die in der Südosttürkei Terror verbreiteten, insbesondere indem sie mit militanten rechten Gruppen kooperierten. ${ }^{21}$

Unabhängig davon, ob Gladio oder Ergenekon Produkte fiktionaler Verschwörung oder plausible Realitätskonstruktionen sind: In der Serie werden die komplexen Beziehungen nicht weiter erläutert. Stattdessen steht Gladio für den Ursprung allen Übels und ist somit eine Variante des Ergenekon-Netzwerks, mit dem es in der Art der Vorgehensweise und in seinen Strukturen stark übereinstimmt. Allerdings unterscheiden die Macher der Serie darin, wie Gladio seine kriminellen Machenschaften begründet von der Art und Weise der Berechtigung, die für Ergenekon angenommen wird. So steht Gladio - aus nationalistischer Perspektive für die Bedrohung der Türkei von außen und stellt einen »verlängerten Arm der Amerikaner « dar. Gladio ist der Feind, der ins Innere eingedrungen ist und von jedem Patrioten - also auch denen, die Teil des Ergenekon-Netzwerks sind - beseitigt werden müsste.

\subsection{Neo-Nationalismus und Ergenekon in Fiktion und Realität}

Neben dem »tiefen Staat « ist der türkische Neo-Nationalismus (ulusalculık) ${ }^{22}$ oder - präziser und im Sinne der Story und ihrer Macher - die Vaterlandsliebe ein Kernthema der Serie. Denn die Hauptmotivation für das engagierte Handeln Polat Alemdars und anderer Agenten ist ihre Vaterlandsliebe. Eine Szene, die die Bedeutung des real existierenden türkischen Nationalismus für das Handeln der

20 Vgl. Ganser 2005.

21 Vgl. Jenkins 2009, S. 44. Eine eindeutige und ausschließliche Erklärung Ergenekons mit den stay behind-operations ist jedoch nach derzeitigem Forschungsstand nicht möglich. Vgl. Kaya 2009, S. $105 \mathrm{f}$.

22 Mit dem Begriff ulusalculik wird eine Fusion linkspolitischer und kemalistisch-nationalistischer Ideologie bezeichnet, die sich vom staatlichen Nationalismus kemalistischer Prägung durch ihre antiimperialistische (und somit dem Westen entgegengesetzte) Haltung unterscheidet. Vgl. Uslu 2008. 
fiktiven Akteure verdeutlicht, zeigt Polats erste Begegnung mit »Gladio « in der dritten Staffel von »Kurtlar Vadisi Pusu «.

Darin nehmen die »KGT «-Agenten zwei Männer fest, die zuvor mit ihrem Hauptgegner İskender Büyük in Kontakt standen. Über diese beiden Männer findet Polat heraus, dass İskender im Dienste "Gladios «steht und auch in deren Auftrag Attentate plant. Um mehr darüber zu erfahren, folgen sie den Hinweisen eines ihrer Gefangenen hin zu einem Buchladen, in dessen Hinterzimmern ein ganzes Team von Agenten an Schreibtischen und Computern arbeitet, um umfangreiche Dossiers anzufertigen. In den Regalen an den Wänden befinden sich Ordner mit den Aufschriften »Kurden-Report«, »Aleviten-Report «, »Eurasien « und »Präsidentenamt «. Doch noch vor dem Buchladen wird das Feuer auf Polat und seine Männer eröffnet. Während sie versuchen, sich in Schutz zu bringen, stehen sie unter Beschuss eines Scharfschützen und können nur rückseits entkommen. Bei ihren Nachforschungen kommen Polat und seine Leute dahinter, dass »Gladio « eine Säuberungsaktion hat durchführen lassen. »Gladio « hat sich ihnen zwar offenbart und seine Existenz und Macht demonstriert, Polat und seine Männer sollen jedoch vorerst keine weiteren Informationen von Belang ergattern können. ${ }^{23}$

Diese Schießerei im Buchladen erinnert an einen weiteren realen Vorfall, der wie Susurluk 1996 auf die Existenz des »tiefen Staates « hinwies, hier aber mit Blick auf die Bedeutung realer nationalistischer Diskurse in der Türkei von Belang ist: 2005 wurde in Şemdinli, einer Kleinstadt im Südosten der Türkei, ein Buchladen in die Luft gesprengt. Der Inhaber des Ladens, Seferi Yılmaz, war ein ehemaliges PKK-Mitglied, das früher wegen terroristischer Aktivitäten verurteilt worden war, die Täter - wie sich später herausstellte - waren Mitglieder der türkischen Gendarmerie. Während der Untersuchungen zum Fall stellte der Staatsanwalt immer mehr Verbindungen zwischen hochrangigen Generälen und dem Vorfall her, doch die eigentlichen Drahtzieher und ihre Intentionen blieben im Dunkeln. ${ }^{24}$

Vergleicht man nun die Interpretation, die »Kurtlar Vadisi Pusu « für Şemdinli anbietet, so steht der Buchladen mit seinen Aktivitäten statt für den realen kurdischen Separatismus und Terrorismus der PKK für Machenschaften der hinter "Gladio "stehenden Amerikaner; mehr noch, die Sprengung des Buchladens selbst ist ebenfalls als Säuberungsaktion der Amerikaner zu sehen. In dieser Interpretation der Vorfälle hat die politische Lage in der Türkei nichts mit dem Vorfall zu tun, stattdessen ist die Türkei ein Spielball externer Mächte - eine verschwörungstheoretisch angeleitete Sicht, die gerade unter nationalistischen Kreisen in

23 »Kurtlar Vadisi Pusu«, 3. Staffel 9. Oktober 2008 bis 11. Juni 2009, Episode 61, Zeit: 49:0050:10.

24 Vgl. Kaya 2009, S. 103. 
der Türkei weit verbreitet ist. ${ }^{25}$ Die Forderungen der Kurden nach bürgerlichen Existenzrechten und die Machenschaften der PKK (sowie deren klandestinen Beziehungen zu staatlichen Organen) werden nicht thematisiert, geschweigedenn differenziert.

Nach dem Anschlag auf den Buchladen rätseln Polat und seine Weggefährten über die Ziele der Amerikaner und ihre nächste Aktion. Doch noch bevor sie sich darüber klar werden können, was die Amerikaner im Schilde führen, wird am Ende der Staffel ein Anschlag auf den türkischen Staatspräsidenten verübt, der sich zum Gebet in einer Moschee befindet. Auch dieses Attentat ist kein Phantasiebild der Drehbuchautoren, sondern findet seine Entsprechung in der Realität. Das bekannteste Beispiel für einen solchen Putschplan veröffentlichte das Nachrichtenmagazin Nokta Anfang 2007 mit den Memoiren von Özden Örnek, einem pensionierten Offizier der Marine. Seine Tagebücher enthielten detaillierte Skizzen zu Putschplänen für den Zeitraum 2004-2005. ${ }^{26}$ Diese Pläne scheiterten zwar offenbar an Widerstand innerhalb der türkischen Armee, dennoch zeigten sie das nationalistische Denken und die umstürzlerischen Absichten eines Teils des türkischen Militärs. Die Coups sollten keine rein militärischen Unternehmungen sein, sondern bezogen Medien, Gewerkschaften, nationalistische Vereine und Universitäten - kurzum: schnell mobilisierbare Teile der Gesellschaft - mit ein. In einer ersten Phase sollten zunächst Studenten und andere Bürger zu Demonstrationen angestachelt werden, danach sollte durch terroristische Angriffe auf Prominente, durch Sabotageakte und Desinformationskampagnen ein öffentliches Klima entstehen, in dem ein Eingreifen des Militärs gerechtfertigt werden sollte. ${ }^{27}$

Auch dieser Skandal, der unter dem Namen Balyoz (Vorschlaghammer) in die jüngere politische Geschichte der Türkei einging, offenbarte die tiefenstaatlichen Strukturen und neo-nationalistischen Ideologien der Ergenekon-Verschwörer, die um jeden Preis die laizistisch-nationalistische Grundordnung der türkischen Re-

25 Vgl. Uslu 2008 sowie zum so genannten "Sèvres-Syndrom «, wonach die in der Türkei von den Nationalisten geschürte Angst vor ausländischen Mächten dem aufoktroyierten Vertrag von Sèvres 1919 entstammt, Jung 2001.

26 Vgl. Kavakci 2009, S. 91, Akbulut 2009, S. 26. Die Ermittlungen um die Tagebücher des Offiziers verliefen jedoch im Sand und der Misserfolg bei der Verfolgung der Şemdinli-Affäre entmutigte die Staatsanwaltschaft die Vorgänge weiter zu hinterfragen. Stattdessen wurde unter dem Vorwand, Nokta habe den Artikel 318 des Strafgesetzbuches verletzt und versucht, vom Militärdienst abzuschrecken, eine Untersuchung gegen Nokta selbst eingeleitet und die Redaktion 2007 geschlossen. Erst im Juni 2007, als Handgranaten in der Wohnung des pensionierten Generals Oktay Yıldırım im Stadtteil Ümraniye in Istanbul gefunden wurden und der Vorfall an die Öffentlichkeit gelangte, wurden weitere Untersuchungen eingeleitet und das Ergenekon-Netzwerk von Teilen der Ermittlungsbehörden und der Medien aufgedeckt. Vgl. auch Grigoriadis, Özer 2010, S. $107 \mathrm{f}$.

27 Vgl. Akbulut 2009, S. 26, Schweers, Osiewicz 2008, S. 68. 
publik - und damit ihre Machtpositionen - aufrecht erhalten wollen. ${ }^{28}$ In diesem Sinn sehen Grigoriadis und Özer diese Strukturen und Ideologien, die auch in der Gesellschaft verwurzelt sind, als Nährboden für das Ergenekon-Netzwerk:

"Named after the mythical location of the birth of the Turkish nation, Ergenekon has a membership base consisting of followers of both nationalist and ulusalc1 groups: retired military and police officers, intellectuals and lawyers, as well as rogue elements. Although a direct relationship between the Ergenekon organization and the ulusalc1 movement cannot be substantiated, Ergenekon is a product of the ideological fermentation in which ulusalcı associations have played a catalytic role. The list of Ergenekon defendants ranged across the Turkish political spectrum from left to right. In addition, the very same rallying points - anti-imperialism, fear of globalization, fear of disintegration and mistrust of the AKP - that brought together the Red Apple Coalition appear to have constituted the reasons for the establishment of the Ergenekon group. ${ }^{29}$

Im weiteren analytischen Nachgang zu Ergenekon gilt demnach auch, dass die neo-nationalistische Bewegung auf einem als kızl elma (roter Apfel) bezeichneten Schulterschluss politisch rechts- und linksgerichteter Kräfte basiert. Im Diskurs der Nationalisten soll der Bruch zwischen der Rechten und der Linken in der Türkei ein vom Ausland hintertriebenes Phänomen sein, das nach dem Ende des Kalten Krieges im Sinne einer freien und unabhängigen Türkei neu reflektiert wurde. Hatten die USA und die Sowjetunion früher Unfrieden zwischen Rechten und Linken gestiftet, so mussten beide Gruppen dagegen den Schulterschluss vollziehen und sich gemeinsam für das türkische Vaterland einsetzen. Diese Allianz konnte vor allem dadurch zu Stande kommen, dass die Linke nach dem Kalten Krieg nach einer neuen Orientierung suchte und nun statt des Gestaltungszieles einer kommunistischen Ordnung den Anti-Imperialismus als Kontrastrategie für sich entdeckte. Im Engagement gegen Globalisierung und Kapitalismus geriet sie dabei bald in ein nationalistisches Fahrwasser. ${ }^{30}$

In der Serie lässt sich dieser Schulterschluss dergestalt nachvollziehen, dass İskender Büyük, eigentlich »Gladio«-Mitglied und Feind der »KGT «, beginnt die Machenschaften »Gladios « immer mehr zu durchschauen. Ihm wird bewusst, wie er und seine Vaterlandsliebe, in deren Namen er zu handeln meinte, für die Zwecke »Gladios « - und damit für die ausländischen Mächte - instrumentalisiert werden. In einer Schlüsselszene sieht sich Polat Alemdar allerdings zunächst kei-

28 Vgl. Girgoriadis, Özer 2009, S. 108, Altan 2012.

29 Girgoriadis, Özer 2009, S. 108.

30 Vgl. ebd., S. $104 \mathrm{f}$. 
neswegs geneigt, ein Bündnis mit İskender Büyük einzugehen und betrachtet ihn nach wie vor als einen seiner Feinde:

Polat: »Var mi öyle şey [...] bir ömür adamlara hizmet et neymiş, kulanıllmış mış. Dün kullanılan, bugünde kullanılır, Memati yarında. İskenderin onlardan olup olmamasının benim gözümde hiçbir değeri yok. [...] « ${ }^{31}$

(Polat: »Gibt es denn so etwas[...]? Ein Leben lang diesen Männern zu Diensten sein und es dann >benutzt werden « nennen. Der, der gestern benutzt wurde, wird heute benutzt und auch morgen. In meinen Augen macht es keinen Unterschied, ob İskender einer von ihnen ist oder nicht. «)

Doch nachdem sich İskender Büyük von "Gladio « gelöst hat, entstehen immer häufiger Situationen, in denen die beiden zusammen gegen den nun gemeinsamen Feind antreten - der Schulterschluss guter Kräfte gegen das externe Böse ist vollzogen. Trotzdem bleibt ein gradueller Unterschied zwischen den "guten « Akteuren bestehen: So wird İskender trotz seines Sinneswandels wegen all der Gewalt und Abscheulichkeiten, die er Polat und seinen Männern angetan hatte (Polat selbst wird von ihm gefangen genommen und wochenlang gefoltert, Hikmet verliert bei einem Angriff İskenders seine Frau und Erhan einen Arm), meist als ein Mann »ohne Werte« präsentiert. Das von den Produzenten gezeichnete Bild İskenders entspricht somit jenen skrupellosen Neo-Nationalisten, die meinen, auf der » richtigen « Seite zu stehen. Dagegen ist Polats Selbstverständnis bzw. das von ihm gezeichnete Bild, dasjenige eines nationalen Helden in moral(istisch)er Reinform. Denn sein Nationalismus bzw. seine Vaterlandesliebe ist eine, die eng mit der islamischen Tradition der Türkei verbunden ist.

So kann mit Blick auf die reale Şemdinli-Affäre und die Aufdeckung des Balyoz-Skandals bzw. des Ergenekon-Netzwerks festgehalten werden, dass diese zwar in der Serie paraphrasiert und realistisch rekonstruiert werden. Die Rekonstruktion und Interpretation der Realität durch die Macher der Serie ist jedoch vereinfacht und in eine bestimmte Richtung gelenkt, die dem Zuschauer den Blick auf andere, realistische Wahrnehmungen versperrt. Putschpläne und gezielte Morde werden ebenso wenig als illegale Machenschaften klandestiner Gruppen kritisch hinterfragt wie die instrumentelle Unterdrückung von Minderheiten und die Frage kurdischer Autonomie- und Unabhängigkeitsbestrebungen. Stattdessen steht »Gladio« allein für den äußeren Feind, der im Innern gegen die nationale

31 »Kurtlar Vadisi Pusu«, 3. Staffel 9. Oktober 2008 bis 11. Juni 2009, Episode 61, Zeit: 55:13$55: 33$. 
Einheit von Staat und Gesellschaft agiert. Gegen diesen müssen sich alle »vaterlandstreuen « Kräfte im Land vereinigen.

\subsection{Das Bild vom Staat: statisch-fiktional vs. dynamisch-real}

Vor dem Hintergrund der Ergenekon-Affäre und den zunehmenden Debatten über Verschwörungen und Putschpläne, lässt sich die vergangene Popularität der Serie "Kurtlar Vadisi Pusu « gut nachvollziehen: Denn die eingebauten, stark an die politische Realität in der Türkei erinnernden Charaktere und Stories schienen die Zuschauer zu fesseln und gaben ihnen die Möglichkeit, die komplexen Skandale und widersprüchlichen Entwicklungen dieser politischen Realität mit Hilfe der einfach gestrickten Interpretationshilfen der Serie zu verstehen. Trotzdem konnte ich in meinen Interviews feststellen, dass das Interesse der Zuschauer an der Serie im Laufe der Zeit zum Teil stark abgenommen hatte. Um die Gründe dafür verstehen zu können, gilt es, die Verschränktheit von Fiktion und Realität auf eine grundsätzliche Ebene zu heben. Die Rolle verschwörerischen Denkens und das Verständnis vom Staat, das in der Serie transportiert wird, berühren diese verschränkte Fiktion-Realität-Beziehung zentral. Um diese nun näher betrachten zu können, ist es wichtig zu fragen, welches Verständnis vom Staat in der Wirklichkeit verhandelt wird und wie die Menschen in der Türkei sich zum Staat positionieren. Einen ersten Anhaltspunkt dafür gibt die Wahrnehmung der ErgenekonAffäre selbst.

2009, nach rund zwei Jahren intransparenter Ermittlungen und rund ein Jahr nachdem vor einem Istanbuler Gericht Anklage gegen hunderte Personen, darunter Ex-Militärs, Politiker, Rechtsanwälte, Geschäftsleute und Journalisten, erhoben worden war, beschrieb Cornell die Lage um die Ergenekon-Prozesse als eine »zwischen Fakten und Fantasie « ${ }^{32}$ :

»Two years since its inception, the Ergenekon case has mushroomed beyond all expectations. In over a dozen predominantly pre-dawn raids, hundreds of suspects have been detained and/or questioned, and almost two hundred have been charged. [...] But far from convincing its critics, the Ergenekon investigation has become ever more

32 Noch heute kann kaum eine andere Einschätzung zu dem Verfahren möglich sein. Noch immer wird intransparent ermittelt, werden Verdächtige zu Dutzenden festgenommen und vor Gerichte gestellt und Prozesse geführt, die kafkaeske Züge aufweisen, ohne Klarheit in die tatsächlichen Machenschaften des kriminellen Netzwerks und des »tiefen Staates « zu geben. Vgl. dazu auch »Ergenekon wird juristisch nicht sauber zu Ende gebracht «, Interview mit Heinz Kramer. Deutsch-Türkische Nachrichten, 15. Mai 2011. 
controversial. On the one hand, it has clearly uncovered information on wrongdoing on the part of some of the accused and certainly on the prevalence of democratically questionable views among a section of the Turkish elite. But that said, the prosecution appears to have failed to live up to the high judicial standards that Turkey's population were entitled to expect, leading to serious doubts concerning the investigation's conduct, and ultimately, its motives. ${ }^{33}$

Angesichts der schwierigen Ermittlungen und der Tatsache, dass die Ergenekon-Prozesse nun schon mehrere Jahre andauern, aber noch zu keinem überzeugenden Ergebnis gelangt sind, ist der Gedanke, dass sie während dieser Zeit verschwörungstheoretisches Denken anregten, alles andere als abwegig. Diese kurzschlussartige Verbindung zwischen Fiktion und Realität oder besser zwischen Fiktion und realistischer Suggestion, wie sie Verschwörungstheorien in der Regel stricken, und wie sie sowohl in den Köpfen der Beobachtern der Ergenekon-Affäre als auch der Zuschauer von »Kurtlar Vadisi Pusu « entsteht, ist nach Žižek als ein Zeichen des Schwindens und Versagens der großen Ideologien zu verstehen:

"In unserem Zeitalter, in dem - in der Politik und der Ideologie wie in der Literatur und im Film - globale umfassende Erzählungen à la >der Kampf der liberalen Demokratie gegen den Totalitarismus zige Möglichkeit, zu einer Art globalem >kognitiven Koordinatensystem ‘ zu gelangen, die paranoide Erzählung einer >Verschwörungstheorie`. [...] Solche Erzählungen dienen [...] als eine Art gleitender Signifikant, den sich verschiedene politische Gruppierungen aneignen können und der ihnen ein minimales kognitives Koordinatensystem verschafft. Dies gilt nicht nur für den Rechtspopulismus und den Fundamentalismus, sondern auch für die liberale Mitte $[\ldots] . \ll^{34}$

Doch so grundsätzlich richtig Žižeks metatheoretische Interpretation erscheint, so wenig (oder so einfach) lässt sie sich auf den konkreten empirischen Fall anwenden. Denn betrachtet man etwa die politischen Ereignisse zum Zeitpunkt des Beginns der Ergenekon-Affäre und die damals ausgestrahlten Teile der Serie, so scheinen die Menschen sich wenig auf Verschwörungstheorien bezogen zu haben. Vielmehr scheint sich zunächst eine große Unsicherheit ob der Komplexität der Thematik breit gemacht zu haben. Mit dem Beginn der Ergenekon-Prozesse 2008, zahllosen Inhaftierungen, Prozessen und sich überschlagenden Meldungen über Komplotte und Verschwörungen war den meisten Menschen nicht klar, gegen wen sich die Verhaftungen richteten, wer in diesem »Spiel « nun gut oder böse,

33 Cornell 2009: vii.

34 Žižek 1999, S. 160. 
wer Gewinner und wer Verlierer war. Für die türkische Bevölkerung führten die Ereignisse seit 2008 und die Unklarheit über Akteure, Absichten, Strukturen und Zusammenhänge vor allem zu wachsender Unsicherheit über die politische $\mathrm{Zu}$ kunft ihres Landes, über die Frage, wer das Land tatsächlich regierte. Richtete sich der Aktionismus der Staatsanwälte und Ermittlungsrichter etwa gegen den vermeintlichen Einfluss der USA im Land? Oder, angestiftet durch die Regierung, gegen das Militär? Hatte die Regierung die Kontrolle über die sich immer schneller entwickelnden Ereignisse verloren? Oder handelte es sich um innenpolitische Machtspiele, die von außen kaum verständlich sein konnten, da notwendige Informationen über das who is who in diesem »Spiel « fehlten und man nicht sicher sein konnte, welche Akteure welche Rollen übernommen hatten?

Zentral wird damit, was im metatheoretischen Sinne Žižeks wie auch im empirischen Fall der Ergenekon-Affäre und ihrer Wahrnehmung, von oberster Relevanz ist: nämlich die Frage, welche Vorstellung vom Staat die Menschen haben und wie sie sich zu ihm in einer Zeit wachsender Komplexität und Unsicherheit positionieren. Dabei kann mit Abrams gesprochen werden, wonach der türkische Fall als ein Paradebeispiel für die von ihm beschriebene Loslösung der (fiktionalen, suggestiven) Idee des Staates von der (reellen und essenziellen) politischen Praxis erscheint:

»The state is not the reality which stands behind the mask of political practice. It is itself the mask which prevents our seeing political practice as it is (...). The state comes into being as a structuration within political practice; it starts its life as an implicit construct; it is then reified - as the res publica, the public reflection no less - and acquires an overt symbolic identity progressively divorced from practice as an illusory account of practice. $\ll^{35}$

Der Staat ist also die Maske, die die politische Praxis bis zu dem Grade unkenntlich macht, dass die Bürger sich fragen müssen, wer eigentlich die Macht hat, der Staat selbst oder geheime, undurchschaubare Kräfte. Wenn reale politische Praxis und die Idee des Staates zu weit auseinander driften, dann kommt der »tiefe Staat « ins Spiel und die politische Praxis wird nicht mehr mit dem institutionalisierten Staat in Verbindung gebracht, sondern ist in der Wahrnehmung der Bürger nur noch eine Farce. Somit wird offensichtlich, dass auch aus Sicht der Beobachter der Ergenekon-Affäre bzw. der Zuschauer von »Kurtlar Vadisi Pusu « der Staat in Fiktion wie in Realität nur mehr zu einer Farce geworden war, eine Verschränkung, die zum Zeitpunkt als die Affäre nach und nach ans Tageslicht kam und sich zeitlich mit der Ausstrahlung der Serie überschnitt, jenes verschwörungs- 
theoretisches Denken über den »tiefen Staat « beförderte, das zu einer weiteren Distanzierung der Bürger von ihrem Staat führte. So nahm die Wahrnehmung des Staates als nicht mehr zuzuordnende Einheit in den Herbst- und Wintermonaten 2008 stetig zu.

Viele meiner Interviewpartner bestätigten damals, dass sie überhaupt nicht mehr verstünden, was während der Ergenekon-Prozesse tatsächlich verhandelt würde und falls man Partei ergreifen wollte, für wen man im Zweifelsfalle eintreten sollte. Die undurchsichtigen politischen Umstände 2008/2009 beförderten das Interesse an "Kurtlar Vadisi Pusu « und waren vermutlich im Sinne der PanafilmProduzenten, die ihre religiös-nationalistische Weltsicht faszinierten Zuschauern darbieten konnten. Im Nachhinein wird jedoch auch deutlich, dass die Produzenten offenbar weder mit der Dynamik, mit der die politische Realität voranschritt, gerechnet hatten, noch mit der Dynamik, mit der sich die Wahrnehmung der Menschen wieder gewandelt hatte.

Denn schon ein Jahr später erschienen den Menschen die einfachen PanafilmInterpretation der politischen Ereignisse unzureichend und stupide. Stattdessen entstand vor allem unter jungen Menschen ab 2009 eine neue Wahrnehmung von Politik. Die Aufdeckung der Ergenekon-Affäre und die damit zusammenhängende Entmachtung des Militärs durch die zivile und demokratisch legitimierte Regierung hatten ebenso ihren Einfluss auf die Menschen, wie die voranschreitende "demokratischen Öffnung " (»demokratik açılım»), von der auch die Kurden und andere Minderheiten im Land profitieren sollten. Darüber hinaus vermittelte die Arbeit an einer neuen, zivilen und demokratische Verfassung vielen Menschen die Hoffnung auf bleibende Veränderungen. ${ }^{36}$

Gerade im universitären Umfeld entstanden neue studentische Gruppen mit demokratisch-liberalem Anspruch und viele neue zivilgesellschaftliche Organisationen, die sich aktiv am politischen Prozess zu beteiligen begannen. Die Wahrnehmung von Politik und dessen, was man in der Politik und durch politisches Engagement erreichen kann, schien sich fundamental zu wandeln. So sagte mir 2010 eine Interviewpartnerin, dass die Zeiten der Militärschläge vorbei seien, die Menschen nun erwacht seien und derartiges nicht mehr zulassen würden. Weitere Interviewpartner lösten sich explizit von Verschwörungstheorien und einer von ihnen fügte hinzu, es sei ihm unbegreiflich, wie man an Verschwörungstheorien glauben könne, jetzt, wo »die Wahrheit ans Licht « gekommen sei und reale politische Ereignisse viel spannender und zugleich gefährlicher für das alte politische Establishment geworden seien als jede Verschwörungstheorie.

36 Vgl. im Allgemeinen Kramer 2009 sowie speziell zur »demokratischen Öffnung « in der Kurdenpolitik Aytaç 2010, Ulusoy 2010. 
Damit lässt sich festhalten, dass die veränderte politische Situation zu einem Wandel des Verständnisses vom Staat geführt hat, aber auch zu der Erkenntnis der Bürger, dass sie die Politik beeinflussen können, dass die Verschwörungstheorien, die ihr Bild vom Staate zuvor geprägt hatten, sie in eine Passivität gedrängt hatten, in die sie sich nicht wieder würden zurückdrängen lassen. Es ist diese Veränderung der Wahrnehmung der Menschen und Zuschauer, die offenbar dazu geführt hatte, dass "Kurtlar Vadisi Pusu « im Lauf der Jahre einen Teil seiner Popularität eingebüßt hatte: So teilten mir verschiedene Interviewpartner $2010 \mathrm{mit}$, dass sie die Serie inzwischen nur noch sporadisch verfolgen würden. Die Macher würden tatsächlich nur noch Mist erzählen - »saçmalıyorlar«.

\section{Ausblick: Die Türkei zwischen Verschwörungsdenken und Demokratisierung}

Die Existenz des »tiefen Staates « erschwert nicht nur eine Analyse politischer Prozesse in der Türkei, sondern ist auch der Faktor, der lange eine Demokratisierung des Landes behindert hat. In Anbetracht eines »tiefen Staates « sind jegliche Versuche politische Prozesse demokratisch beeinflussen zu wollen, zum Scheitern verurteilt. Wie im Rahmen dieses Essays gezeigt werden konnte, gilt, dass es offensichtlich egal ist, ob man nun die Fiktion einer TV-Action-Serie oder die politischen Realität betrachtet: Der »tiefe Staat « will Unklarheit, Unsicherheit, Angst und Schrecken verbreiten und die Bürger effektiv davon abhalten, kritisch und aktiv am politischen Leben teilzunehmen. In tiefenstaatlichen Strukturen können Geheimagenten wie die »KGT « in »Kurtlar Vadisi Pusu « oder selbsternannte »Retter « der staatlichen »Ordnung « wie die kriminellen Verschwörer des Ergenekon-Netzwerkes ungehindert agieren. Den Bürgern selbst wird dagegen jegliche Initiative, sich aktiv einzubringen und politisch zu engagieren, genommen. Gingeras' Verständnis des »tiefen Staates « legt sogar im Sinne Žižeks nahe, dass diese Entmündigung der Bürger die Hauptfunktion des »tiefen Staates « sein könnte, da er ein Produkt des paranoiden Sicherheitsdenkens moderner Nationalstaaten sei. Dieses Sicherheitsdenken weist auf die innere Schwäche des Staates selbst hin, der nur in Koalition mit der Mafia und dem Militär gegen das Volk zu überleben vermag. Seine Möglichkeiten innerhalb legaler Strukturen zu operieren, sind so stark beschränkt, dass er diese Allianzen braucht. ${ }^{37}$

Bemerkenswert ist, dass sich die tiefstaatlichen Strukturen in der Türkei nie gänzlich verselbstständigt haben, sondern - wie auch die Geheimagenten in »Kurtlar Vadisi Pusu" - immer bestimmten Idealen der etablierten kemalisti- 
schen Ordnung treu geblieben sind. ${ }^{38}$ Mit dem Aufstieg und der Machtkonsolidierung der AKP wird allerdings eine "postkemalistische Republik « nun immer wahrscheinlicher, die keinen Raum mehr für alte, nationalistische Ideale zu lassen scheint. Die Frage, die sich dabei allerdings stellt, ist, wohin der Weg dieser postkemalistischen Türkei führt. Für einige Analysten ist die AKP eine konservativ-demokratische Partei und die Zukunft der Türkei eine demokratische. ${ }^{39}$ Diese Wahrnehmung wird jedoch von anderen nach wie vor in Zweifel gezogen. So sehen Rubin und Cağaptay die AKP als eine islamistische Partei, die die Türkei zunehmend islamisieren will. ${ }^{40}$

Beide Standpunkte sind in Bezug auf jüngste Entwicklungen allerdings zu einfach gefasst. Mit Sicherheit lässt sich jedoch sagen, dass die hoffnungsvolle Politik der AKP, die von ihr angestrebte demokratische Öffnung sowie die Arbeit an einer neuen Verfassung von den Ergenekon-Prozessen und deren Aus- und Nachwirkungen überschattet werden. Die Tatsache, dass die Verhaftungen im Rahmen der Prozesse jedes Ausmaß sprengen und für die Bürger undurchsichtig und nicht mehr nachvollziehbar sind, hat viele Erwartungen für mehr Demokratie und Rechtstaatlichkeit enttäuscht. Anstelle eines wachsenden Vertrauens in die Politik, wie sie 2010 noch spürbar war, ist Skepsis zurückgekehrt und vielen erscheint es, als wolle die AKP alle ihre Kritiker inhaftieren lassen. ${ }^{41}$

Nichtsdestotrotz kann jedoch auch rückblickend festgehalten werden, dass die Entwicklungen bis 2011 in Richtung Demokratisierung und Partizipation gewiesen haben. In Hinblick darauf, dass meine Gespräche mit jungen türkischen Studenten 2010 auf ein stärker werdendes Interesse an Politik und eine aktive Auseinandersetzung mit dem Staat hinwiesen, lässt sich behaupten, dass eine autoritäre, islamistische Ausrichtung der türkischen Republik (von der etwa Rubin und Çağaptay ausgehen) nicht im Sinne dieser Bevölkerungsgruppe ist und auf starken Widerstand stoßen könnte. So ist auch vielen der Studenten durchaus bewusst, dass Premierminister Erdoğan kein reformeifriger Demokrat ist, sondern eher ein neo-liberaler Konservativer, der zu wenig bewegt. ${ }^{42}$ In diesem Sinne ge-

38 Nach Meinung mancher gilt das bis heute auch für das türkische Militär. So weisen etwa Schweers und Osiewicz darauf hin, dass das Militär in der Türkei niemals eine dauerhafte Militärdiktatur angestrebt hat, sondern sich traditionell der gesamtstaatlichen, nationalen Einheit und dem kemalistischen Prinzip des Laizismus verschrieben hatte. (Vgl. Schweers, Osiewicz 2008, S. 75.) Doch muss auch betont werden, dass, selbst wenn das Militär als Garant für die Stabilität und nationale Einheit des Landes unter kemalistischen Idealen aufgetreten ist, so hat es bislang stets aber auch einen »unmündigen Bürger « favorisiert.

39 Vgl. beispielsweise Hale und Özbudun 2010, Kavakci, 2009.

40 Vgl. beispielsweise Rubin 2012, Cağaptay 2009.

41 Dazu und zur Polarisierung der türkischen Gesellschaft in zwei Lager pro und kontra zur Regierung und deren »Enthüllungspolitik« vgl. Kramer 2009b, Gürsoy 2012.

42 Vgl. Jung 2011, Cizre, Walker 2010. 
hen für viele die Reform- und Demokratisierungsbemühungen seit $2010 \mathrm{zu}$ langsam, die Stagnation seit dem Verfassungsreferendum ist für Liberale als auch für Muslime mehr als unbefriedigend. Dennoch spricht einiges für die von Jung geteilte Erwartung, dass die einzige Möglichkeit für eine stärkere Demokratisierung bei der AKP selbst liegt: »Mit seiner dritten Amtszeit und der anstehenden Ausarbeitung einer neuen Verfassung hat Erdoğan die Möglichkeit, die Türkei in eine demokratische und pluralistische Zukunft zu führen. Die kommenden Jahre werden zeigen, ob die AKP dazu in der Lage ist. « ${ }^{43}$

\section{Quellen- und Literaturverzeichnis}

Abrams, P. 1988 (1977). Notes on the Difficulty of Studying the State. Journal of Historical Sociology 1 (1): 58-89.

Akbulut, H. 2009. Die zivilmilitärischen Beziehungen in der Türkei: zwischen Putschbestrebungen und Demokratisierungsbemühungen. Österreichisches Institut für Internationale Politik, Arbeitspapier 6o, Wien. http://www.oiip.ac.at/fileadmin/Unterlagen/ Dateien/Publikationen/Die_zivil-militaerischen_Beziehungen.pdf. Zugegriffen: 05. Oktober 2012.

Altıparmak, O., Berlinski, C. 2011. The Wikileaks Cables on Turkey: 20/20 Tunnel Vision. Middle East Review of International Affairs (MERIA) 15 (1). http://www.gloria-center. org/2011/o8/meria20103the-wikileaks-cables-on-turkey-2020-tunnel-vision/. Zugegriffen: 05. Oktober 2012.

Aytaç, Ö. 2010. The Democratic Initiative and the Kurdish Issue in Turkey since 2009. Turkish Policy Quarterly 9 (1): 101-116.

Cağaptay, S. 2009. Free media will save Turkish democracy. Blog Middle East Strategy at Harvard, 29. September 2009. https://blogs.law.harvard.edu/mesh/2009/o9/freemedia-will-save-turkish-democracy/. Zugegriffen: 5. Dezember 2012.

Cizre, U., Walker, J. 2010. Conceiving the New Turkey after Ergenekon. The International Spectator 23 (1): 89-98.

Cornell, S. E. 2009. Preface. In Between Fact and Fantasy - Turkey's Ergenekon Investigation. Silk Road Paper, Washington, hrsg. Jenkins, G. H. http://www.silkroadstudies. org/new/docs/silkroadpapers/o9o8Ergenekon.pdf. Zugegriffen: 5. Dezember 2012.

Ganser, D. 2005. Terrorism in Western Europe: An Approach to NATO's Secret Stay-Behind Armies. In The Whitehead Journal of Diplomacy and International Relations 6 (1): 69-97.

Gingeras, R. 2010. Last Rites for a >Pure Bandit «: Clandestine Service, Historiography and the Origins of the Turkish Deep State. Past and Present 206 (1): 151-174.

Grigoriadis, I. N., Özer, I. 2010. Mutations of Turkish Nationalism: From Neo-Nationalism to the Ergenekon Affair. Middle East Policy 17 (4): 101-113.

43 Jung 2011, S. 6. 
Gürsoy, Y. 2012. Public Attitudes toward the Military and Ergenekon Case: Consequences for the Consolidation of Democracy in Turkey. Istanbul Bilgi University European Institute, Working Paper 5, http://eu.bilgi.edu.tr/images/pictures/working_paper_5.pdf. Zugegriffen: 5. Dezember 2012.

Hale, W., Özbudun, E. 2010. Islamism, Democracy and Liberalism in Turkey. The Case of the $A K P$. London et al.: Routledge.

Hermann, R. 2008. Wohin geht die türkische Gesellschaft? Kulturkampf in der Türkei. München: dtv.

Jenkins, G. H. 2009: Between Fact and Fantasy - Turkey's Ergenekon Investigation, Silk Road Paper, Washington, http://www.silkroadstudies.org/new/docs/silkroadpapers/ 0908Ergenekon.pdf. Zugegriffen: 5. Dezember 2012.

Jung, D. 2001. >The Sèvres Syndrome «: Turkish Foreign Policy and its Historical Legacy. In

Oil and Water: Co-operative Security in the Persian Gulf, hrsg. Møller, B., 131-159. London: I. B. Tauris.

Jung, D. 2011. Die Türkei auf dem Weg in eine neue Republik? Die Türkei nach dem Rücktritt des Generalstabs. GIGA Focus Nahost 8. Hamburg.

Kaya, S. 2009. The Rise and Decline of the Turkish >Deep State $<$ The Ergenekon Case. Insight Turkey 11 (4): 99-113.

Kavakci, M. 2009. Turkey's Test with its Deep State. Mediterranean Quarterly 20 (4): 83-97.

Kramer, H. 2009a. Mutige Öffnung in der türkischen Innen- und Außenpolitik. SWP Aktuell 54, Berlin.

Kramer, H. 2009b. Türkische Turbulenzen: der andauernde Kulturkampf um die »richtige« Republik. SWP-Studie 11, Berlin.

Schweers, P., Osiewicz, P. 2008. »Der Hüter Republik - Zur Rolle des Militärs in der modernen Türkei «. WeltTrends 16 (5): 68-76.

Ulusoy, K. 2010. The >democratic opening « in Turkey: a historical/comparative perspective. Insight Turkey 12 (2): 71-90.

Uslu, E. 2008. Ulusalcılık: The Neo-nationalist Resurgence in Turkey. Turkish Studies 9 (1): 73-97.

Žižek, S. 1999. Liebe deinen Nächsten? Nein, Danke! - Die Sackgasse des Sozialen in der Postmoderne. Berlin: Volk und Welt.

\section{Nachrichten- und weitere Internetquellen}

Altan, A. 2012. Balyoz ve Avrupa. Taraf Online, 25. September 2012. http://taraf.com.tr/ahmet-altan/makale-balyoz-ve-avrupa.htm. Zugegriffen: 5. Dezember 2012.

»Ergenekon wird juristisch nicht sauber zu Ende gebracht. Interview mit Heinz Kramer«. Deutsch-Türkische Nachrichten, 15. Mai 2011. http://www.deutsch-tuerkische-nachrichten.de/2011/05/88595/. Zugegriffen: 5. Dezember 2012.

Kurt, N. 2004. Kurtlar Dergahı. Hürriyet Magazin, 25. Juni 2004. http://arama.hurriyet.com. tr/arsivnews.aspx?id=236217. Zugegriffen: 5. Dezember 2012.

Kürsü, S. 2007. Kurtlar Vadisi sansürü Ehud Olmert'e jest mi? Anafikir.com, 17. Februar 2007. http://www.anafikir.com/kurtlar-vadisi-sansuru-ehud-olmerte-jest-mi/. Zugegriffen: 5. Dezember 2012. 
Özcan, B. 2009. Onu hiç böyle görmediniz!, Pazar Vatan Online, 19. April 2009. http://pazarvatan.gazetevatan.com/haberdetay.asp?hkat=1\&hid=13765\&yaz=G\%FCncel. Zugegriffen: 5. Dezember 2012.

Özüzcan, Ş. 2009. Muro'nun en büyük korkusu. Bugün, 05. September 2009. http://www. bugun.com.tr/haber-detay/73436-muro-nun-en-buyuk-korkusu-haberi.aspx. Zugegriffen: 5. Dezember 2012.

"Necati Şaşmaz Vatan'a konuştu«. Vatan, 20. April 2009. http://www.yazete.com/magazin/ necati-sasmaz-vatan-a-konustu-8712.html. Zugegriffen: 5. Dezember 2012.

Panafilm 2009. Kamuoyuna Duyurulur. http://www.kurtlarvadisi.com/index.php? site= news\&news_id=277. Zugegriffen: 23. Dezember 2012)

»Polat Alemdar Suriye'de fenomen oldu «. Habertürk, 19. September 2012. http://www.haberturk.com/medya/haber/777677-polat-alemdar-suriyede-fenomen-oldu, Zugegriffen: 5. Dezember 2012.

Rebhandl, B. 2010. Wir haben ein Mandat in Palästina. FAZ.net, 3. November 2010. http:// www.faz.net/aktuell/feuilleton/medien/2.1756/tuerkei-wir-haben-ein-mandat-inpalaestina-11056555.html. Zugegriffen: 5. Dezember 2012.

Rubin, B. 2012. This week, Turkey went a long way toward becoming an Islamic Republic. The Rubin Report, 7. Februar 2012. http://rubinreports.blogspot.de/2012/o6/thisweek-turkey-went-long-way-toward.html. Zugegriffen: 5. Dezember 2012.

Satmış, D. 2004: Kurtlar Vadisi'nde 3 doğru 1 yanlış. Hürriyet, 12. Juli 2004. http://arama. hurriyet.com.tr/arsivnews.aspx?id=240479. Zugegriffen: 5 . Dezember 2012.

»TV-Serien: Neue diplomatische Kraft der Türkei «. Deutsch-Türkische Nachrichten, 12. Dezember 2011. http://www.deutsch-tuerkische-nachrichten.de/2011/12/12249/tv-serien-neue-diplomatische-kraft-der-tuerkei/. Zugegriffen: 5. Dezember 2012.

Open Access. This chapter is distributed under the terms of the Creative Commons Attribution Noncommercial License, which permits any noncommercial use, distribution, and reproduction in any medium, provided the original author(s) and source are credited. 